\title{
Spatial information of fuzzy clustering based mean best artificial bee colony algorithm for phantom brain image segmentation
}

\author{
Waleed Alomoush ${ }^{1}$, Ayat Alrosan², Ammar Almomani ${ }^{3}$, Khalid Alissa $^{4}$, Osama A. Khashan ${ }^{5}$, \\ Ahmad Al-Nawasrah6 \\ ${ }^{1}$ Computer Department, Imam Abdulrahman Bin Faisal University, Dammam, Saudi Arabia \\ ${ }^{2}$ Deanship of Information and Communication Technology, Imam Abdulrahman Bin Faisal University, Dammam, \\ Saudi Arabia \\ ${ }^{3}$ Department of Information Technology, Al-Huson University College, Al-Balqa Applied University, Irbid, Jordan \\ ${ }^{4}$ Department of Computer Science, College of Computer Science and Information Technology, Imam Abdulrahman \\ bin Faisal University, Dammam, Saudi Arabia \\ ${ }^{5}$ College of Computing and Informatics, Saudi Electronic University, Riyadh, Saudi Arabia \\ ${ }^{6}$ Information and Communication Technology College, British university of Bahrain, Bahrain
}

\begin{tabular}{l} 
Article Info \\
\hline Article history: \\
Received Dec 18, 2020 \\
Revised Mar 5, 2021 \\
Accepted Mar 16, 2021 \\
\hline Keywords: \\
Artificial bee colony \\
Clustering algorithms \\
Global best \\
Optimization algorithms \\
Phantom image
\end{tabular}

Phantom image

\begin{abstract}
Fuzzy c-means algorithm (FCM) is among the most commonly used in the medical image segmentation process. Nevertheless, the traditional FCM clustering approach has been several weaknesses such as noise sensitivity and stuck in local optimum, due to FCM hasn't able to consider the information of contextual. To solve FCM problems, this paper presented spatial information of fuzzy clustering-based mean best artificial bee colony algorithm, which is called SFCM-MeanABC. This proposed approach is used contextual information in the spatial fuzzy clustering algorithm to reduce sensitivity to noise and its used MeanABC capability of balancing between exploration and exploitation that is explore the positive and negative directions in search space to find the best solutions, which leads to avoiding stuck in a local optimum. The experiments are carried out on two kinds of brain images the Phantom MRI brain image with a different level of noise and simulated image. The performance of the SFCM-MeanABC approach shows promising results compared with SFCM-ABC and other stats of the arts.
\end{abstract}

This is an open access article under the CC BY-SA license.

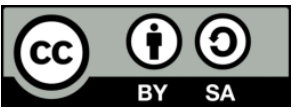

\section{Corresponding Author:}

Waleed Alomoush

Computer Department

Imam Abdulrahman Bin Faisal University

Dammam, Saudi Arabia

Email: wkalomoush@iau.edu.sa

\section{INTRODUCTION}

Segmentation of Image is a method that divides a raw image into a group of regions without overlapping, and each region has distinctive aspects to obtain certain interesting objects. Conventional image segmentation methods contain region extraction, edge detection, clustering-based, and histogram method (threshold). Segmentation of medical image is a process to determine the region of interest (RIO), for instance, structures of anatomical and anomalies such as tumor and cyst. Also, for medical usages like planning of therapeutics, diagnostics, and guidance [1]-[3]. Methods of image segmentation were merging 
with several current computation models, including the clustering approach, wavelet analysis, and evolutionary algorithms, which have gained significant attention medical image applications has been restriction, because the problems of overlapping between regions and homogeneities-based intensity contrast limitations and resolution, which were led to a complex process of segmentation. Fuzzy set theory was proposed to avoid this challenge; with a goal to enable partial membership classification using an objective membership feature. Fuzzy clustering methods are used as a segmentation method, which is most commonly implemented and used effectively in the segmentation of images [4]-[6]. Fuzzy c-means algorithm (FCM) is among the most frequently used clustering algorithms. The FCM is based on the repeat optimization of the fuzzy fitness function. Because it has high performance in the computational process, effectiveness and straight forward, it is commonly used in the clustering of image. In contrast, it has serious shortcomings such as sensitivity to noise and stuck in local optimum [7], [8]. In order to solve these problems, evolutionary algorithms and variants such as firefly algorithm (FA) [9], [10], particle swarm optimization (PSO) [11], cuckoo search (CS) [12], [13], and artificial bee colony (ABC) [14] and harmony search (HS) [15]-[17] have been successes in many domains such as segmentation of images [1], [2], [8], [18]-[20], clustering [21] and in several fields [22], [23]. In this paper, several studies have been reviewed, which are related to image segmentation methods based clustering approach with evolutionary algorithms, these studies are reviewed as follow, In Alrosan et al. [20], proposed fuzzy c-means were combined with ABC algorithms and called ABC-FCM, and this method was carried out by two kinds of MRI images namely the simulated brain MRI images, Alrosan et al. [8], [24] presented a novel version of ABC algorithm, namely (Mean WBC) that is merged with the FCM clustering approach. This method was conducted on real brain MRI images. Bose and Mali [5] proposed an image segmentation method known as FABC, in this study the conventional FCM is merged with the ABC algorithm. The membership fitness function of fuzzy-type 2 is used, where $\mathrm{ABC}$ has been used to find centroids location. Relevantly, Ouadfel and Meshoul [25] presented FCM based on a modified ABC, namely MoABC-FCM. The outcomes of the proposed approach show that promising when compared to other related works. Salima et al. [26] introduced a fuzzy clustering method based on the ABC algorithm, called ABC-SFCM. This research has been applied to medical, synthetic, and real images. Furthermore, Hancer et al. [27] proposed an image segmentation method using hydride ABC to extract brain tumours from the MRI brain image.

Mekhmoukh and Mokrani [28] presented a new technique for image segmentation including the particle swarm optimization (PSO) algorithm and collaboration between both the outer rejection and the set of a level. The findings have been contrasted with many related works approaches, and it has been more efficient, where it's high computational time. An automatic clustering K-means algorithm, namely DCPSO was presented by [29]-[31]. In addition, an optimum number of clusters have been selected through the use of binary PSO and different cluster validity indices, the value of the objective function for measurement of the evolving process. And then, centroids of the selected clusters were defined using the K-means approach. So this paper applied the approach for segmenting multispectral, real, synthetic images.

Additionally, Alia et al. [7] Developed a novel dynamic fuzzy clustering technique (DC) and used the FCM hybrid harmony search (HS) algorithm to generate an automatic segmentation methodology named DCHS for actual and simulated MRI brain images. Meanwhile, Zhang et al. [32] Presented the FCM combination with biogeography-based optimization (BBO) with a different version of BBO algorithms. The experiments were tested on groups of natural images. Also, Alomoush et al. [18] introduced an unsupervised clustering algorithm based hybrid fuzzy c-means algorithm with a firefly algorithm by (FA), namely FFCM. The proposed method was tested by sets of real MRI brain image (IBSR) and simulated brain image (SBD). The evaluation process of FFCM proved promising outcomes when compared with FCM and other related works. in contrast, the FFCM was stilling has limitations such as sensitivity to noise [19].

Based on above mentioned studies, many of these approaches are stuck in local optima, can't solve overlapping problem between regions in images segmented and are very sensitive to noise. Accordingly, the proposed method (SFCM-MeanABC) is developed to solve or reduce these limitations using contextual information in the spatial fuzzy clustering algorithm to reduce sensitivity to noise and its used MeanABC capability of balancing between exploration and exploitation that is explore the positive and negative directions in search space to find the best solutions (location of centroids), which leads to avoiding stuck in a local optimum. This paper is represented in six sections: the second is the spatial FCM algorithm, the original $\mathrm{ABC}$ and MeanABC are represented in section three, the proposed of SFCM-MeanABC is presented in section four, in section five the experiments are discussed, finally, the conclusion is presented in Section six.

\section{THE SPATIAL FCM ALGORITHM}

Salima et al. [26] presented SFCM clustering algorithm to compute the local spatial correlation between neighboring pixels by reformulated the membership function of the standard FCM algorithm using 
spatial information. The new fuzzy membership function of SFCM is computed in (1) and the centers of clusters determined in (2).

$$
\begin{gathered}
U_{i k}^{*}=\frac{\sum_{j \in N_{k}} u_{i j} p_{k j}}{\sum_{s=1}^{c} \sum_{j \in N_{k}} u_{s j} p_{k j}} \\
V_{i}^{*}=\frac{\sum_{k=1}^{n} u_{i k}^{* m} x_{k}}{\sum_{k=1}^{n} u_{i k}^{* m}}
\end{gathered}
$$

Where $U_{i k}$ is the membership function value in the conventional FCM. The probability $p_{k j}$ to find the influence of the adjacent pixels on the central pixel $X_{k}$ and exploits the spatial information. $N_{k}$ stands for the elements of adjacent locating into an around the central pixel $X_{k}$. the interaction between elements is increased when the nearest adjacent has similar features and the influence is strong, the $P_{k j}$ of the neighbor $X_{j}$ is based on two factors: the pixels intensity or feature attraction $d_{k j}$ and the spatial positions of the neighbors or distance attraction $d^{s}{ }_{k j}$. The feature attraction $d_{k j}^{f}$ is defined as the absolute intensity difference between the pixel and its neighbors and it is defined in (3):

$$
d_{k j}^{f}=\left|x_{k}-x_{j}\right|
$$

And the distance attraction $d^{s}{ }_{k j}$ is defined as the Euclidean distance between the coordinates of the pixel and its neighbors as shown in (4) and the factor $P k j$ is defined as shown in (5):

$$
\begin{aligned}
& d_{k j}^{s}=\|k-j\|^{2} \\
& P_{k j}=\frac{1}{d_{k j}^{f} / \sigma_{k}^{2}} X \frac{1}{d_{k j}^{s}}
\end{aligned}
$$

Where $\sigma_{k}^{2}$ represents the local density around of the central pixel $X_{k}$. The probability $P_{k j}$ increases when the grey level of the nearest $j^{\text {th }}$ neighbours is close to the grey level of the central pixel $X_{k}$. The new objective function of SFCM algorithm is computed in (6):

$$
J_{F C M}^{*}\left(U^{*}, V\right)=\sum_{k=1}^{n} \sum_{t=1}^{c} u_{i k}^{* m} d^{2}\left(x_{k}, v_{i}\right)
$$

\section{THE ORIGINAL ABC AND MEANABC ALGORITHMS}

The standard of $\mathrm{ABC}$ algorithm was presented by Karaboga and Basturk [33], that is inspired using swarms bee of behavior. The ABC and MeanABC are explained.

\subsection{The artificial bee colony (ABC)}

The bees into ABC algorithm are classified into three types: the kind of employed bees that is focus on finding sources of food and sending their search information to the kind bee of onlookers. Then, the onlooker bees retrieve searches to carry out better solutions based on the food sources quality which is discovered by employ. The ABC algorithm randomly initialized the source of food as in (7).

$$
x_{i, j}=x_{m i n, j}+\operatorname{rand}(O, 1)\left(x_{\max , j}-x_{\min , j}\right)
$$


Where $(i=1, \ldots, S N)$ and $S N$ are indicated to the solutions $D$ of parameters value $(j=1,2, \ldots, D)$, while $x j$ min and $x j$ max are the upper and lower value of the dimension $j$, respectively. The value of the updated source of food is computed as in (8).

$$
v_{i j}=x_{i j}+\phi_{i j}\left(x_{i j}-x_{k j}\right)
$$

Where: $K \in(1,2, \ldots S N)$ and $j \in(1,2, \ldots D)$ indices, which are randomly selected and $K \neq i . \emptyset_{i j}$ is value from $[-1,1]$. The value of fitness function $f(x i)$ that is calculated using (9). That kind of onlooker bee chose solutions with certainty, that is considered the correlation exists between fitness value of a source of food and the employed. The probability of fitness is computed as in (10),

$$
\begin{aligned}
& \text { fit }\left(x_{i}\right)=\left\{\begin{array}{cc}
\frac{1}{\left(1+f\left(x_{i}\right)\right)} & i F f\left(x_{i}\right) \geq 0 \\
1+a b s\left(f\left(x_{i}\right)\right) & i F f\left(x_{i}\right)<0
\end{array}\right. \\
& p^{i}=\frac{f i t_{i}}{\sum_{n=1}^{s n} f i t_{i}}
\end{aligned}
$$

In this way, when several trials of the solution are same, the employee bees will convert to scout bees and abandon their solutions. Accordingly, (7) shows how the scout bees start new searches and solutions randomly until they reached the criterion of termination.

\subsection{The mean artificial bee colony (MeanABC)}

In Alrosan et al. [8], [24] an improved new version of ABC that is called MeanABC, and it presents a new modified search equation for the kind of employ bee phase, which depends on the information about the best candidates to regenerate a new neighborhood source of food as in (11).

$$
v_{i j}=x_{i j}+\phi_{i j}\left(x_{i j}-x_{k j}\right)+\Psi_{i j}\left[\left(\frac{\text { pbest }_{i}+\text { gbest }}{2}-x_{i j}\right)+\left(\frac{\text { pbest }_{i}-\text { gbest }}{2}-x_{i j}\right)\right]
$$

Where $x i, j$ is the first part of the equation that is indicated to present position, then $\emptyset_{i j}\left(x_{i j}-x_{k j}\right)$ is the difference between the current position and the new position, and $\emptyset_{i j}$ between [-1,1] which is a random value. Part one and two are identical to standard ABC. The third part is the new modified search equation into (MeanABC). Where the first side is very important for switching the present position of employ bee towards the mean best value of the positive way of the best global position.

$$
\left(\frac{\text { pbest }_{i}+\text { gbest }}{2}-x_{i j}\right),\left(\frac{\text { pbest }_{i}{ }^{\text {gbest }}}{2}-x_{i j}\right)
$$

The second side is switching the present mean position of pbest, which is the positive way of own best position and the - gbest negative way of the best global position. The value of $\psi$ between $[0, \mathrm{C}]$, that is a random value, and $C$ is a positive value. The value of $\mathrm{C}$ is constant that plays an important role in balancing between exploration and exploitation.

\section{THE SPATIAL FUZZY CLUSTERING BASED MEAN ARTIFICIAL BEE COLONY ALGORITHM (SFCM-MEANABC)}

The spatial fuzzy clustering algorithm (SFCM) is improved the performance of the image segmentation process with a noisy level. In contrast, SFCM is still trapped into local optimum. So, to avoid stuck into local optimum, this paper proposed a new clustering algorithm for image segmentation based on the MeanABC optimization algorithm, which uses the mean global best searching ability of the MeanABC to search the optimum cluster centers. In the proposed clustering algorithm (SFCM-MeanABC), a swarm of $N_{p}$ bees represents a set of cluster centers. Each single bee $z_{i}$ in set of solutions $G$ is formulated as $Z_{i}=\left\{v_{i l}, v_{i 2}, \ldots, v_{i c}\right\}$ where $v_{i k}$ represents the $k^{\text {th }}$ cluster center for the $i^{\text {th }}$ bee. The solutions quality is computed by the objective function in (12) and (13): 


$$
\begin{aligned}
& f i t_{i}=\frac{1}{1+J_{i}^{*}\left(U^{*}, V\right)} \\
& J_{i}^{*}\left(U^{*}, V\right)=\sum_{j=1}^{c} \sum_{k=1}^{n} u_{j k}^{{ }^{*} m}\left\|x_{k}-v_{i j}\right\|^{2}
\end{aligned}
$$

When the value of $J_{i}^{*}$ is small, that leads to greatest value of fitness equation fiti and the clustering outcomes were improved. When the approach reached to the convergence, the fuzzy partition matrix is converted to matrix of a crisp partition. The defuzzification is come using allocate each pixel to the cluster with the greatest value of membership. The main steps of SFCM-MeanABC algorithm are presented in Figure 1.

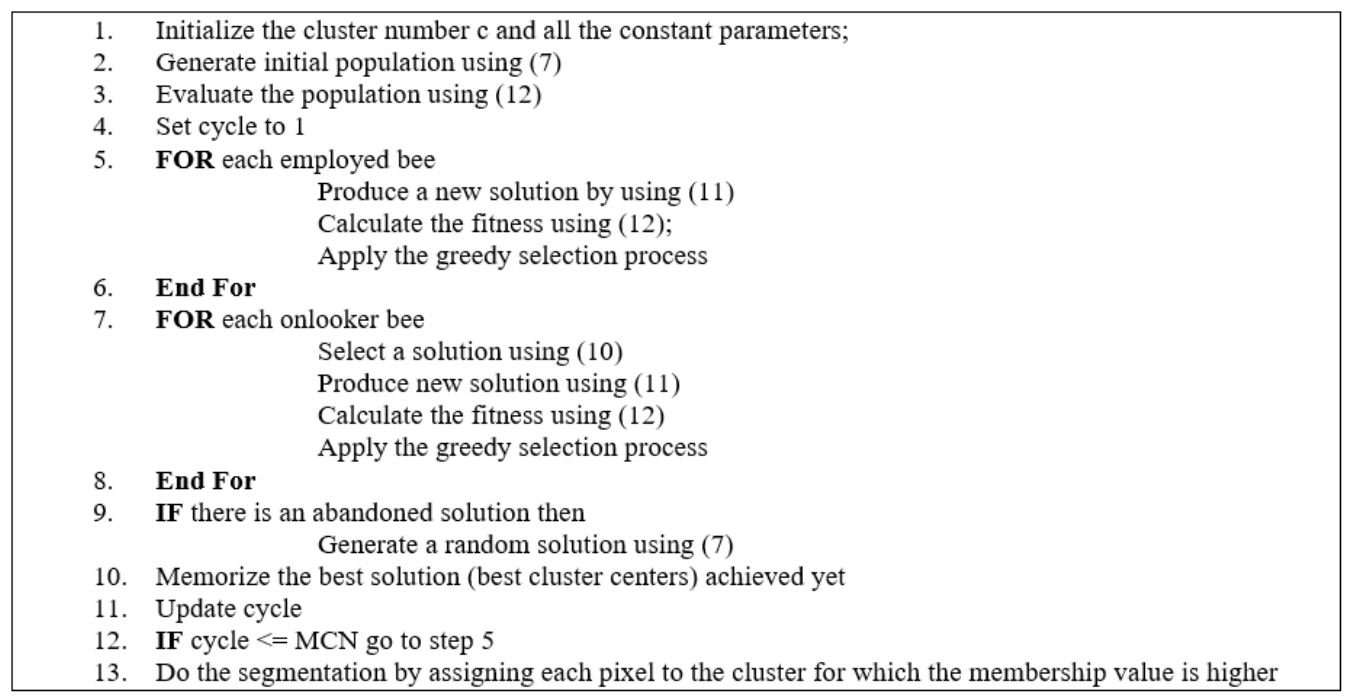

Figure 1. Spatial fuzzy clustering based mean artificial bee colony algorithm (SFCM-MeanABC)

\section{RESULTS AND DISCUSSION}

In this section, the experiments are conducted on the phantom MRI brain image. This section is divided into two parts. The first experiment is carried out on the phantom MRI brain image dataset which is consists of 3\%, \%5 and 7\% noise level, and no intensity in-homogeneity. The second experiment is carried out using simulated brain database (SBD). These images were obtained from the results of a simulated brain dataset performed by McGill University [34]. According to [26, 35], the number of clusters (region) assigned is 4: gray matter (GM), white matter (WM), cerebrospinal fluid (CSF), and background (BKG).

The parameter settings for SFCM-MeanABC follow the parameter setting in [26], where the population size $S N(N p)=20$, maximum cycle number $(M C N)=2000$, weighting exponent $m=2$ nonnegative constant parameter $C=1.43$ and limit $=100$. The accuracy for proposed method SFCM-MeanABC and other related works FCM, FCM_S, GA-FCM, GA-FCM_S, FCMA-ES and FCMA-ES_S [35], also, FCM_S1, FCM_S2, NGFCM, SFCM and ABC-SFCM [26], these algorithms are evaluated by Jaccard similarity $(J)$ where $J$ between $(0,1)$ and the best value for $J$ is the maximum value $=1$. The Jaccard similarity measures the similarity of two sets as the ration of the size of their intersection divided by the size of their union as defined in (19). Let $V_{g}^{k}$ and $V_{s}^{k}$ denote the total number of pixels labeled into a cluster k in the ground truth $(\mathrm{g})$ and the obtained segmentation (s). For cluster k, the Jaccard similarity $J^{k}(g, s)$ is defined by (14):

$$
J^{k}(g, s)=\frac{\left|V_{g}^{k} \frown V_{s}^{k}\right|}{\left|V_{g}^{k} \smile V_{s}^{k}\right|}
$$

A good segmentation is obtained when $J^{k}(g, s)$ is near 1 which means that the cluster $k$ is well detected. 


\subsection{First part}

In this experiment, SFCM-MeanABC is compared with other related works of image segmentation approach like FCM, FCM_S1, FCM_S2, novel generalized FCM (NGFCM), spatial fuzzy c-means (SFCM) and ABC-SFCM [26]. In Table 1, the first column contains the noise level for the image. The second column represents the name of fuzzy clustering approaches as reported in [26] and SFCM-MeanABC. The rest of the columns contain the accuracy values by Jaccard similarity $(J)$ for 4 clusters (CSF, GM, WM, and BKG) respectively with different noise levels. The bold items represent the better outcomes obtained by clustering approaches in this study. Table 1 compares the accuracy and reliability outcomes obtained from the different clustering algorithms: SFCM-MeanABC, FCM, FCM_S1, FCM_S2, NGFCM, SFCM and ABC-SFCM.

Table 1. Jaccard Index for different clustering methods on phantom MR image with different noise levels

\begin{tabular}{cccccc}
\hline Noise level & Method & CSF & GM & WM & BKG \\
\hline $3 \%$ & FCM & 0.80 & 0.86 & 0.80 & 0.92 \\
& FCM_S1 & 0.84 & 0.89 & 0.88 & 0.95 \\
& FCM_S2 & 0.87 & 0.92 & 0.90 & 0.96 \\
NGFCM & 0.90 & 0.94 & 0.91 & 0.96 \\
& SFCM & 0.90 & 0.94 & 0.90 & 0.96 \\
& ABC-SFCM & 0.91 & 0.95 & 0.90 & 0.97 \\
$5 \%$ & SFCM-MeanABC & 0.96 & 0.98 & 0.95 & 0.98 \\
& FCM & 0.73 & 0.85 & 0.75 & 0.91 \\
& FCM_S1 & 0.77 & 0.88 & 0.89 & 0.94 \\
& FCM_S2 & 0.82 & 0.89 & 0.90 & 0.96 \\
& NGFCM & 0.81 & 0.90 & 0.90 & 0.96 \\
& SFCM & 0.83 & 0.91 & 0.91 & 0.96 \\
& ABC-SFCM & 0.84 & 0.90 & 0.92 & 0.97 \\
$7 \%$ & SFCM-MeanABC & 0.93 & 0.95 & 0.94 & 0.98 \\
& FCM & 0.68 & 0.79 & 0.70 & 0.80 \\
& FCM_S1 & 0.72 & 0.88 & 0.80 & 0.93 \\
& FCM_S2 & 0.77 & 0.88 & 0.82 & 0.94 \\
NGFCM & 0.78 & 0.89 & 0.84 & 0.942 \\
& SFCM & 0.79 & 0.90 & 0.86 & 0.942 \\
& ABC-SFCM & 0.79 & 0.90 & 0.86 & 0.946 \\
& SFCM-MeanABC & 0.89 & 0.94 & 0.93 & 0.96 \\
\hline
\end{tabular}

It's clearly shown in Table 1 that SFCM-MeanABC outperforms other clustering algorithms [26]. SFCM-MeanABC has much robustness and stability because the SFCM-MeanABC approach employs the reformulated objective function based SFCM, this leads to the reduction of the sensitivity to noise. Also, SFCM-MeanABC has better accuracy owing to the good ability of MeanABC in balancing between exploration and exploitation. It also has the ability to search in the positive and negative direction in search space, which is why SFCM-MeanABC outperforms other approaches. Figure 2 shows the original image, SFCM-MeanABC outcomes of segmented image, and ground truth image, respectively. In Figure 2, the segmented image by SFCM-MeanABC is much closer to the ground truth because SFCM-MeanABC has the capability to reduce the effectiveness of noise on segmentation accuracy. These capabilities are based on the employment of the reformulated objective function of SFCM and the improvement of the ABC search mechanism.

\subsection{Second part of experiments}

In this experiment, SFCM-MeanABC is compared with other related works of image segmentation approach like FCM, FCM_S, GA-FCM, GA-FCM_S, FCMA-ES and FCMA-ES_S [35]. In Table 2, The first column represents the name of fuzzy clustering approaches as reported in [35] and SFCM-MeanABC. The rest of the columns contain the accuracy values by Jaccard similarity $(J)$ for CSF, GM and WM. The bold items represent the better outcomes obtained by clustering approaches in this study. It's clearly shown in Table 2 that SFCM-MeanABC outperforms other clustering algorithms [35]. SFCM-MeanABC has much robustness and stability because the SFCM-MeanABC approach employs the reformulated objective function based SFCM. Also, SFCM-MeanABC has better accuracy owing to the good ability of MeanABC in balancing between exploration and exploitation. Figure 3 shows the original image, ground truth of WM, GM and CSF, and SFCM-MeanABC outcomes of segmented image of WM, GM and CSF, respectively.

In general, the segmentation outcomes of SFCM-MeanABC for WM, GM and CSF tissues are robust than those of the related works approaches [35], because SFCM-MeanABC has the ability to decrees the sensitive to noise by utilizing the new equation of fitness function of SFCM. Also, most related works approaches have misclassification between CSF, GM and WM tissues based on three causes: first one is the images artifacts affect the levels of intensity images which have overlapping between image segments as in 
brain tissues, secondly the structure complexity of SDB images that have high levels of noise which leads to wrong classification based on other related works approaches.
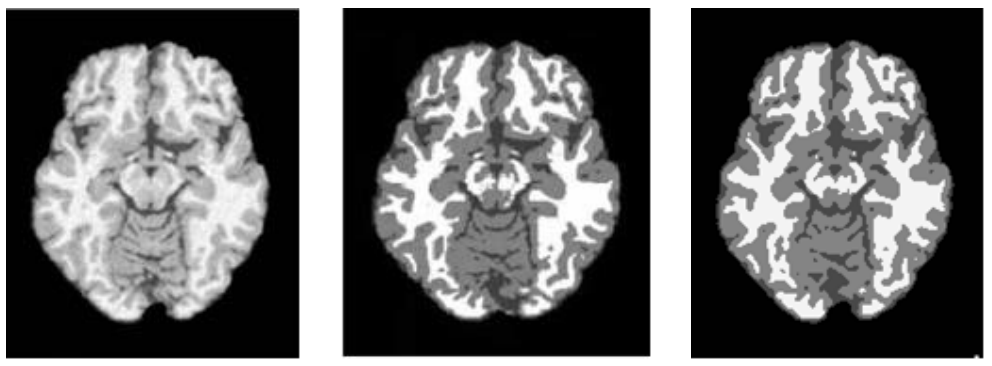

Figure 2. Phantom MR original image, segmented image by SFCM-MeanABC and ground truth image, respectively

Table 2. Jaccard index for different clustering methods

\begin{tabular}{lccc}
\hline Algorithms & CSF & GM & WM \\
\hline SFCM-MeanABC & 0.9520 & 0.9036 & 0.9611 \\
FCMA-ES_S & 0.9114 & 0.8219 & 0.9145 \\
FCMA-ES & 0.9007 & 0.8238 & 0.8965 \\
GA-FCM_S & 0.9038 & 0.8194 & 0.8981 \\
GA-FCM & 0.8982 & 0.8073 & 0.8954 \\
FCM_S & 0.9106 & 0.8242 & 0.9102 \\
FCM & 0.9042 & 0.8021 & 0.8872 \\
\hline
\end{tabular}

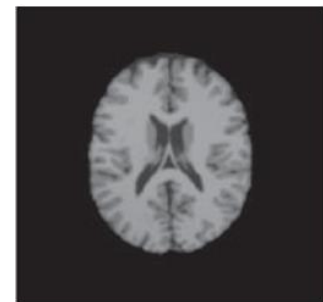

(a)

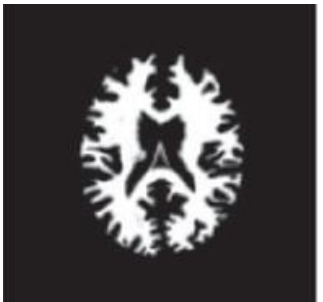

(b)

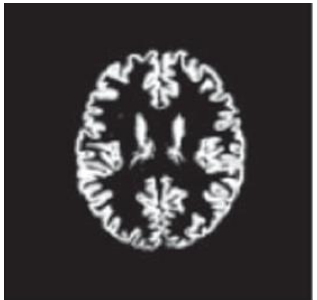

(c)

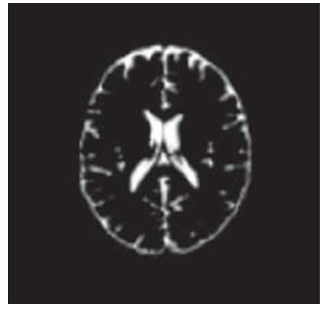

(d)

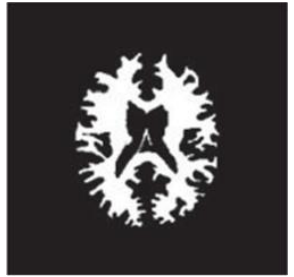

(e)

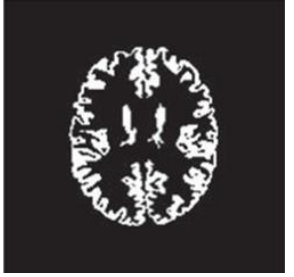

(f)

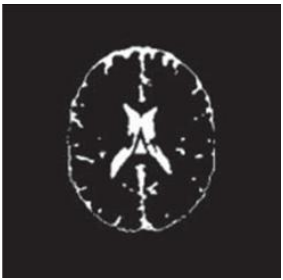

$(\mathrm{g})$

Figure 3. Segmented image by SFCM-MeanABC; (a) original image, (b) ground truth (WM), (c) ground truth (GM), (d) ground truth (CSF), (e) SFCM-MeanABC outcomes (WM), (f) SFCM-MeanABC outcomes (GM), (g) SFCM-MeanABC outcomes (CSF)

\section{CONCLUSION}

In this paper, spatial information of fuzzy clustering-based mean best artificial bee colony algorithm, is presented, SFCM-MeanABC. This proposed approach is used contextual information in the spatial fuzzy clustering algorithm to reduce sensitivity to noise and its used MeanABC capability of balancing between exploration and exploitation that is explore the positive and negative directions in search space to find the best solutions, which leads to avoiding stuck in a local optimum. The experiments are carried out on two kinds of brain data set, the Phantom MRI brain image with a different level of noise and simulated image. The performance of the SFCM-MeanABC approach shows promising results compared with SFCM-ABC 
and other stats of the arts. The future works of this research as follow, firstly, to use different type of fuzzy clustering algorithm based other kind of swarm intelligence algorithm. Secondly, to reduce noise sensitive of fuzzy clustering algorithm using image filtering mechanisms.

\section{ACKNOWLEDGEMENTS}

This research was supported by grant No, 2020-064-PYSS, Date 22/4/2020 which is followed to Imam Abdulrahman Bin Faisal University.

\section{REFERENCES}

[1] Alomoush W. et al., "A Survey: Challenges of Image Segmentation Based Fuzzy C-Means Clustering Algorithm," Journal of Theoretical and Applied Information Technology, vol. 96, no. 16, pp. 5153-5170, 2018.

[2] W. K. Alomoush W. and K. Omar, "Dynamic fuzzy c-mean based firefly photinus search algorithm for MRI brain tumor image segmentation," in PhD, Computer science, Universiti Kebangsaan Malaysia, 2015.

[3] S. N. Kumar, A. L. Fred, H. A. Kumar, and P. S. Varghese, "Firefly Optimization Based Improved Fuzzy Clustering for CT/MR Image Segmentation," in Nature Inspired Optimization Techniques for Image Processing Applications, Springer, Cham, vol. 150, p. 1-28, 2019, doi: 10.1007/978-3-319-96002-9_1.

[4] M. A. Balafar, "Fuzzy C-mean based brain MRI segmentation algorithms," Artificial Intelligence Review, vol. 41, no. 3, pp. 441-449, 2014, doi: 10.1007/s10462-012-9318-2.

[5] A. Bose and K. Mali, "Fuzzy-based artificial bee colony optimization for gray image segmentation," Signal, Image and Video Processing, vol. 10, no. 6, pp. 1089-1096, 2016, doi: 10.1007/s11760-016-0863-z

[6] C. Ozturk, E. Hancer, and D. Karaboga, "Improved clustering criterion for image clustering with artificial bee colony algorithm," Pattern Analysis and Applications, vol. 18, no. 3, pp 587-599, 2015, doi: 10.1007/s10044-0140365-y.

[7] O. M. Alia, R. Mandava, and M. E. Aziz, "A hybrid harmony search algorithm for MRI brain segmentation," Evolutionary Intelligence, vol. 4, no. 1, pp. 31-49, 2011, doi: 10.1007/s12065-011-0048-1.

[8] A. Alrosan, W. Alomoush, N. Norwawi, M. Alswaitti, and S. N. Makhadmeh, "An improved artificial bee colony algorithm based on mean best-guided approach for continuous optimization problems and real brain MRI images segmentation," Neural Computing and Applications, vol. 33, no. 5, pp. 1671-1697, 2020, doi: 10.1007/s00521-02005118-9.

[9] Xin-She Yang, "Firefly algorithm, stochastic test functions and design optimisation," arXiv preprint arXiv:1003.1409, vol. 2, no. 2, pp. 78-84, 2010

[10] W. Alomoush, K. Omar, A. Alrosan, Y. M. Alomari, D. Albashish, A. Almomani, "Firefly photinus search algorithm," Journal of King Saud University - Computer and Information Sciences, vol. 32, no. 5, pp. 599-607, 2020, doi: 10.1016/j.jksuci.2018.06.010

[11] J. Kennedy and R. Eberhart, "Particle swarm optimization," Proceedings of ICNN'95 - International Conference on Neural Networks, Perth, WA, Australia, vol. 4, 1995, pp. 1942-1948, doi: 10.1109/ICNN.1995.488968.

[12] X. Yang and Suash Deb, "Cuckoo Search via Lévy flights," 2009 World Congress on Nature \& Biologically Inspired Computing (NaBIC), Coimbatore, India, 2009, pp. 210-214, doi: 10.1109/NABIC.2009.5393690.

[13] W. Alomoush, "Cuckoo Search Algorithm based Dynamic Parameter Adjustment Mechanism for Solving Global Optimization Problems," International Journal of Applied Engineering Research, vol. 14, no. 24, pp. 4434-4440, 2019.

[14] B. Basturk, "An artificial bee colony (ABC) algorithm for numeric function optimization," in IEEE Swarm Intelligence Symposium, Indianapolis, IN, USA, 2006.

[15] Z. W. Geem, J. H. Kim, and G. V. Loganathan, "A new heuristic optimization algorithm: harmony search," Simulation, vol. 76, no. 2, pp. 60-68, 2001, doi: 10.1177/003754970107600201.

[16] A. A. Alomoush, A. A. Aleswari, H. S. Almari, K. Z. Zamli, W. Alomoush, and M. I. Younis, "Modified Opposition Based Learning to Improve Harmony Search Variants Exploration," in International Conference of Reliable Information and Communication Technology, Springer, Cham, 2019, pp. 279-287, doi: 10.1007/978-3030-33582-3_27.

[17] A. Alomoush, K. Z. Zamli, A. Alrosan, W. Alomoush, K. Alissa, and A. R. Alsewari, "Enhancing three variants of harmony search algorithm for continuous optimization problems," International Journal of Electrical and Computer Engineering (IJECE), vol. 11, no. 3, pp. 2343-2349, 2021, doi: 10.11591/ijece.v11i3.pp2343-2349.

[18] W. Alomoush, S. Abdullah, S. Sahran, and R. I. Hussain, "MRI brain segmentation via hybrid firefly search algorithm," Journal of Theoretical and Applied Information Technology, vol. 61, no. 1, pp. 73-90, 2014.

[19] W. Alomoush, S. N. H. S. Abdullah, S. Sahran, and R. I. Hussain, "Segmentation of MRI brain images using FCM improved by firefly algorithms," Journal of Applied Sciences, vol. 14, no. 1, pp. 66-71, 2014, doi: 10.3923/jas.2014.66.71.

[20] A. Alrosan, N. M. Norwawi, W. Ismail, and W. Alomoush, "Artificial bee colony based fuzzy clustering algorithms for MRI image segmentation," in International Conference on Advances in Computer Science and Electronics Engineering-CSEE, pp. 225-228, 2014, doi: 10.13140/RG.2.1.4671.8326.

[21] Alomoush, W. and A. Alrosan, "Metaheuristic Search-Based Fuzzy Clustering Algorithms," arXiv preprint arXiv: $1802.08729,2018$ 
[22] B. Aldeeb, M. A. AlBetar, A. O. Abdelmajeed, and M. J. Younes, "Comprehensive Review of Uncapacitated University Examination Timetabling Problem," International Journal of Applied Engineering Research, vol. 14, no. 24, pp. 4514-4547, 2019.

[23] M. Alauthman, A. Almomani, M. Alweshah, and W. Alomoush, "Machine Learning for Phishing Detection and Mitigation: Principles, Algorithms, and Practices," Machine Learning for Computer and Cyber Security, vol. 26, pp. 48-74, 2019, doi: 10.1201/9780429504044-2.

[24] A. Alrosan and N. Norwawi, "Mean artificial bee colony optimization algorithm to improve fuzzy c-means clustering technique for gray image segmentation," in PhD, Computer science, Universiti Kebangsaan Malaysia, 2017.

[25] S. Salima and S. Meshoul, "Handling fuzzy image clustering with a modified ABC algorithm," International Journal of Intelligent Systems and Applications, vol. 4, no. 12, pp. 65-74, 2012, doi: 10.5815/ijisa.2012.12.09.

[26] O. Salima, A. T. Ahmed, and B. Mohamed, "Spatial information based image clustering with a swarm approach," IAES International Journal of Artificial Intelligence (IJ-AI), vol. 1, no. 3, pp. 149-160, 2012, doi: 10.11591/ijai.v1i3.765.

[27] E. Hancer, C. Ozturk and D. Karaboga, "Extraction of brain tumors from MRI images with artificial bee colony based segmentation methodology," 2013 8th International Conference on Electrical and Electronics Engineering (ELECO), Bursa, Turkey, 2013, pp. 516-520, doi: 10.1109/ELECO.2013.6713896.

[28] A. Mekhmoukh and K. Mokrani, "Improved Fuzzy C-Means based Particle Swarm Optimization (PSO) initialization and outlier rejection with level set methods for MR brain image segmentation," Computer methods and programs in biomedicine, vol. 122, no. 2, pp. 266-281, 2015, doi: 10.1016/j.cmpb.2015.08.001.

[29] M. Omran, A. Engelbrecht, and A. A. Salman, "Particle swarm optimization methods for pattern recognition and image processing," Swarm Intelligence in Data Mining, vol. 34, 2006.

[30] M. G. H. Omran, A. Salman, and A. P. Engelbrecht, "Dynamic Clustering using Particle Swarm Optimization with Application in Unsupervised Image Classification," in Fifth World Enformatika Conference (ICCI 2005), Prague, Czech Republic, 2005, pp. 199-204.

[31] M. G. H. Omran, A. Salman, and A. P. Engelbrecht, "Dynamic clustering using particle swarm optimization with application in image segmentation," Pattern Analysis and Applications, vol. 8, no. 4, pp. 332-344, 2006, doi: 10.1007/s10044-005-0015-5.

[32] M. Zhang, W. Jiang, X. Zhaou, Y. Xue, and S. Chen, "A hybrid biogeography-based optimization and fuzzy Cmeans algorithm for image segmentation," Soft computing, vol. 23, no. 6, pp. 2033-2046, 2019, doi: 10.1007/s00500-017-2916-9.

[33] D. Karaboga. and B. Basturk, "A powerful and efficient algorithm for numerical function optimization: artificial bee colony (ABC) algorithm," Journal of global optimization, vol. 39, no. 3, pp. 459-471, 2007, doi: 10.1007/s10898-007-9149-x.

[34] BainWeb, "Simulated brain database Mcconnell Brain Imaging Centre Montreal Neurological Institute Mcgill University," [Online]. Available: http://www.bic.mni.mcgill.ca/brainweb

[35] D. Mohammed, S. Mohammed, R. B. Bouiadjra, and M. Rebbah, "CMA-ES based fuzzy clustering approach for MRI images segmentation," International Journal of Computers and Applications, pp. 1-7, 2019. 